\section{Characterization of cytoskeleton features and maturation status of cultured human iPSC-derived cardiomyocytes}

Christian Zuppinger, ${ }^{1}$ George Priyanka Dutta-Passecker, ${ }^{2}$ Adrian Segiser, ${ }^{3}$ Henriette Most, ${ }^{3}$ Thomas M. Suter ${ }^{1}$

${ }^{1}$ Cardiology Department, Bern University Hospital, Switzerland ${ }^{2}$ Axol Bioscience, Cambridge, UK ${ }^{3}$ Cardiovascular Surgery Department, Bern University Hospital, Switzerland

\begin{abstract}
Recent innovations in stem cell technologies and the availability of human induced pluripotent stem cell-derived cardiomyocytes (hiPSC-CMs) have opened new possibilities for studies and drug testing on human cardiomyocytes in vitro. Still, there are concerns about the precise nature of such 'reprogrammed' cells. We have performed an investigation using immunocytochemistry and confocal microscopy on several cellular features using commercially available hiPSC-CMs. For some selected developmentally regulated or cardiac chamber-specific proteins, we have compared the results from hiPSC-derived cardiomyocytes with freshly isolated, ventricular cardiomyocytes from adult rats. The results show that all typical cardiac proteins are expressed in these hiPSC-CMs. Furthermore, intercalated disclike structures, calcium cycling proteins, and myofibrils are present. However, some of these proteins are only known from early developmental stages of the ventricular myocardium or the diseased adult heart. A heterogeneous expression pattern in the cell population was noted for some muscle proteins, such as for myosin light chains, or incomplete organization in sarcomeres, such as for telethonin. These observations indicate that hiPSC-CMs can be considered genuine human cardiomyocytes of an early developmental state. The here described marker proteins of maturation may become instrumental in future studies attempting the improvement of cardiomyocyte in vitro models.
\end{abstract}

\section{Introduction}

There is a demand in the life sciences for a constant supply of highly differentiat- ed cell types such as cardiomyocytes, hepatocytes, endothelial cells, and neuronal cells that often show a limited life span, minimal proliferation capacity, and rapid de-differentiation in primary cell culture. For primary human cardiomyocytes, there is practically no supply since healthy organs are rarely available and the cells quickly expire without perfusion. Embryonic stem cells (ESC) originating from the blastocyst stage of early human embryos raise ethical questions and cannot be obtained from the same patient for cell therapies. The few immortalized cardiomyocyte cell lines that are commercially available are of animal origin and provide little advantage over primary cardiomyocytes from neonatal rodents. However, since the first publication in 2006 with murine and 2007 with human cells by Yamanaka et al., ${ }^{1}$ so-called induced pluripotent stem cells (iPSC) have rapidly become a popular tool. Cells differentiated from iPSC appear as a valid alternative to primary cells for laboratory studies, drug development, and regenerative medicine. In the last few years, there have been considerable advances in the methods of 're-programming' somatic cells to iPSCs using non-viral transfection and genome editing. ${ }^{2}$ Subsequently, these cells can be used to produce differentiated human cell types such as cardiomyocytes (hiPSC-CMs) using established protocols. ${ }^{3,4}$ Existing applications of hiPSC-CMs include drug safety testing, disease modeling, and tissue engineering. ${ }^{2,5}$ With this method, it is possible to obtain cells from adult patients with cardiac disorders, with a well-documented disease and family history, to study the resulting cellular phenotype in detail. Additionally, hiPSC-derived cell types can be genetically modified, for example, to test a gene therapy approach in vitro demonstrating the attempted rescue in a relevant genetic setting. A remaining concern with hiPSC-CMs is their reportedly immature developmental status and diverse phenotypes. ${ }^{6,7}$

We have investigated several cellular features in commercially available hiPSCCMs by immunocytochemistry and confocal microscopy to characterize this type of model system for future use in toxicology and basic research. For some selected proteins, we have compared expression and organization with freshly isolated, adult cardiomyocytes. This study provides a 'snapshot' of several cytoskeleton proteins of this cell type in culture. We did not attempt to improve maturation of the cells outside of culturing for ten days to replicate a typical experimental situation where the non-proliferating cells are used from frozen stock, treated with test compounds, and analyzed.
Correspondence: Dr. Christian Zuppinger, Cardiology Department, Bern University Hospital, MEM G803b, Murtenstrasse 35, CH-3008 Bern, Switzerland.

Tel. +41316328699 - Fax: +41316323297

E-mail: christian.zuppinger@dkf.unibe.ch

Key words: Cardiomyocytes; heart; confocal microscopy; hiPSC; sarcomere; development; muscle.

Contributions: $\mathrm{CZ}$, research conception and design; $\mathrm{CZ}, \mathrm{AS}, \mathrm{GG}$, performing experiments; PDP, HM, TMS, interpretation of experimental results and critical revision of the manuscript.

Conflict of interest: GG and PDP are employees of Axol Bioscience.

Acknowledgements: We thank Prof. Hugues Abriel and the channelopathies group (University of Bern, Switzerland) for valuable advice and support. We thank Dr. Irina Agarkova (Federal Institute of Technology Zurich) for antibodies to embryonic heart myomesin and to M-protein. This study was performed with the support of the Microscopy Imaging Center (MIC), University of Bern.

Funding was provided by the Swiss Heart Foundation.

Received for publication: 20 December 2016 Accepted for publication: 7 June 2017.

This work is licensed under a Creative Commons Attribution-NonCommercial 4.0 International License (CC BY-NC 4.0).

(C) Copyright C. Zuppinger et al., 2017 Licensee PAGEPress, Italy

European Journal of Histochemistry 2017; 61:2763 doi:10.4081/ejh.2017.2763

\section{Materials and Methods}

\section{Cell culture}

Human iPSC-derived cardiomyocytes (hiPSC-CMs, ax2505) were obtained from Axol Bioscience (Little Chesterford, Cambridge, UK). The original hiPSC cell line was produced using an episomal vector and CD34+ cord blood cells from a male newborn donor. Cryopreserved cardiomyocytes were rapidly thawed, then diluted in maintenance medium with supplements (Axol Bioscience, ax2530) and 10\% fetal bovine serum. The cells were then seeded into dishes with a glass-bottom well of 10 $\mathrm{mm}$ diameter (MatTek, Ashland, MA, USA) coated with $1 \%$ gelatin in water (SigmaAldrich, St. Louis, MO, USA; G1393). After $48 \mathrm{~h}$, plating medium was exchanged 
with maintenance medium without serum for the first time, and then changed every two days.

\section{Immunocytochemistry of cultured} cells and confocal microscopy

Cells cultured for 10 days on glass-bottom dishes (MatTek) were washed with phosphate buffered saline (PBS) then fixed with 3\% para-formaldehyde in PBS for 15 min, permeabilized with $0.2 \%$ Triton-X100 (Sigma-Aldrich) in PBS for $10 \mathrm{~min}$, incubated for $30 \mathrm{~min}$ with bovine serum albumin (Sigma-Aldrich) $1 \mathrm{mg} / \mathrm{mL}$ in PBS at room temperature, incubated overnight with primary antibodies at $4^{\circ} \mathrm{C}$, washed 3 times with PBS and incubated for $1 \mathrm{~h}$ with secondary antibodies goat anti-rabbit or goat antimouse coupled to AlexaFluor488 or AlexaFluor546 fluorescent dyes (Thermofisher Scientific/Molecular Probes, Waltham, MA, USA) at room temperature in the dark. Primary antibodies used in this study are listed in Table 1. Antibodies to embryonic heart myomesin (EH-myomesin) and M-protein were a kind gift from Dr. Irina Agarkova (Federal Institute of Technology, ETHZ, Zurich, Switzerland). Preparations were embedded using non-hardening solutions with anti-fading protection with or without DAPI (SlowFade Diamond, Invitrogen, Carlsbad, CA, USA; or Vectashield/DAPI, Vector Laboratories, Burlingame, CA, USA). Preparations were examined with a Zeiss LSM 710 confocal microscope using EC Plan-Neofluar 40x/1.30 and Plan-Apochromat $60 \mathrm{x} / 1.40$ oil immersion lenses (Carl Zeiss, Oberkochen, Germany). Manual counting of the respective fraction of cells showing protein expression and sarcomeric organization was conducted using at least 200 cells from multiple fields of confocal images from 3 independent immunocytochemistry experiments and different batches of hiPSC-CMs. Measurement of morphologic features was performed using the software NIH-ImageJ (U.S. National Institutes of Health and available on the Internet at http://rsb. info.nih.gov/nihimage/) on confocal images obtained from cardiomyocytes immunostained for the sarcomeric M-line marker myomesin. A lineprofile was created along the myofibril and distances between peaks were measured. Only cells at resting length were evaluated. For the form factor, maximum lengths and widths of cells in low-density cultures were measured and expressed as a ratio. At least ten images showing several cells were used for the analysis of each feature and the data were statistically analyzed using the twotailed Student's -test (Graphpad Prism).
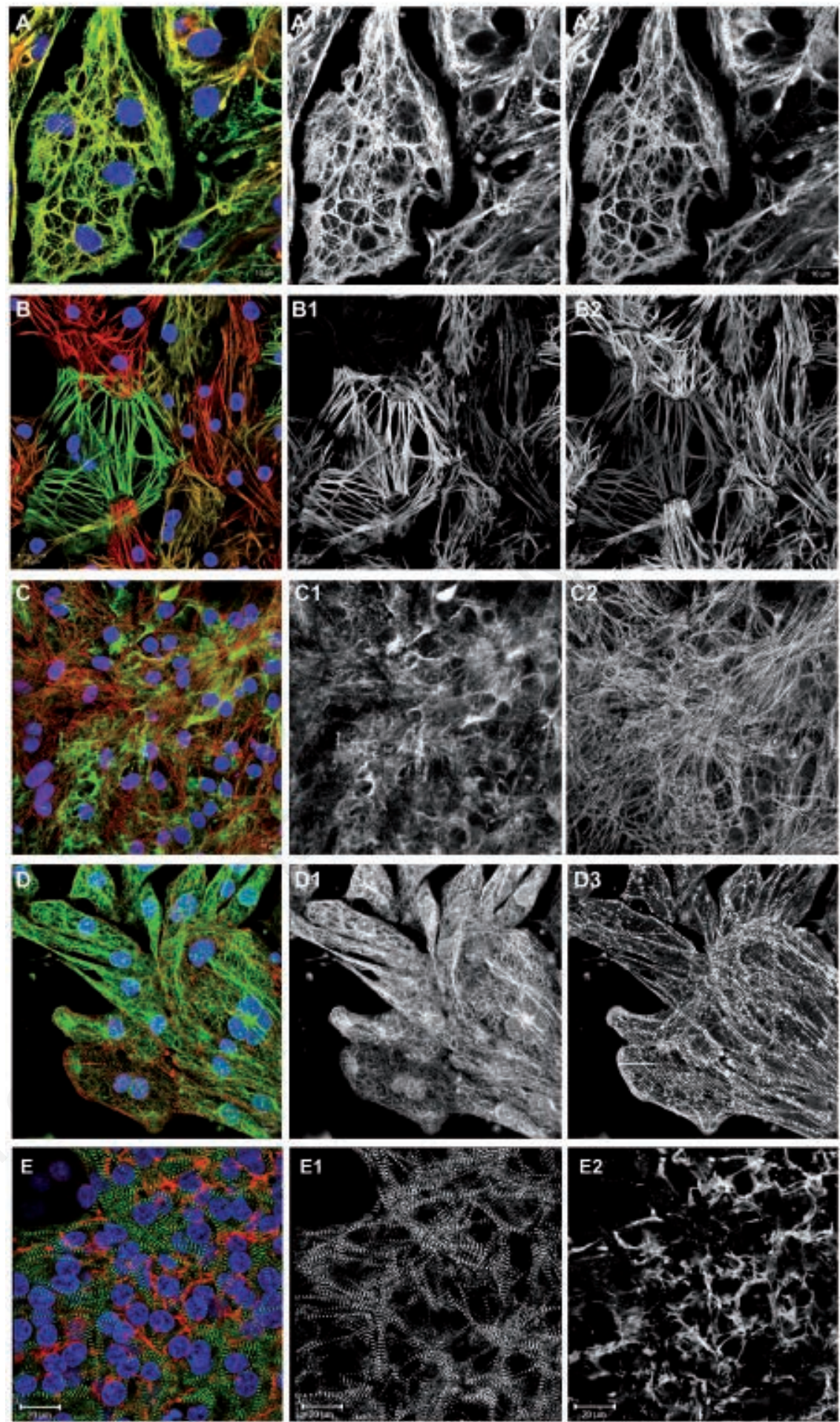

Figure 1. Cytoskeleton and sarcomeric proteins. Overlay images including DAPI are shown on the left, followed by corresponding green and red channel images in greyscale. A) Cells were immunostained for alpha-MHC (green), beta-MHC (red) and DNA (blue). B) Cells were immunostained for MLC2v (green), MLC2a (red) and DNA (blue). Cells expressing both isoforms appear in orange-yellow. C) Cells were immunostained for vimentin (green), myomesin (red) and DNA (blue). D) Cells were immunostained for alpha-tubulin (green), sarcomeric alpha-actinin (red) and DNA (blue). E) Cells were immunostained for myomesin (green), laminin (red) and DNA (blue). 


\section{Isolation of adult rat cardiomy- ocytes and immunocytochemistry}

Adult rat ventricular cardiomyocytes (ARVM) were isolated as previously described $^{8,9}$ from 3 months old (350-400 g) male Wistar rats, seeded onto gelatine-coated chamber slides and immediately fixed with 4\% p-formaldehyde in PBS for 20 minutes. The preparations were permeabilized with 1\% Triton-X100 (Sigma-Aldrich) in PBS for $20 \mathrm{~min}$, incubated for $1 \mathrm{~h}$ with bovine serum albumin (Sigma-Aldrich) 1 $\mathrm{mg} / \mathrm{mL}$ in PBS at room temperature, incubated overnight with primary antibodies at $4^{\circ} \mathrm{C}$, washed 3 times with PBS and incubated $2 \mathrm{~h}$ with secondary antibodies coupled to Alexa fluorescent dyes (Thermofisher/ Molecular Probes) at room temperature. Preparations were then washed, embedded and examined as described for the hiPSCCMs. Only primary antibodies equally recognizing both human and rodent protein isoforms were selected for this experiment. All experiments were carried out according to the Swiss animal protection law and with the permission of the canton Bern veterinary office (license BE32/14). This investigation conforms to the Guide for the Care and Use of Laboratory Animals published by the US National Institutes of Health (NIH Publication No. 85-23, revised 1996).

\section{Results}

Cytoskeleton and sarcomeric proteins

Cryopreserved hiPSC-CMs were thawed and cultured for ten days as described in the materials and methods section. The cells were then fixed and immunostained for several cytoskeleton and sarcomeric proteins (Figure 1). In several co-stainings, a well-described marker of the sarcomeric M-line, myomesin, ${ }^{10,11}$ served to demonstrate cellular identity and myofibrillar content in the cultured cells (Figure 1 C2, E1 and Figure 2 C2,D2,E2). Essential muscle proteins comprise the family of myosin heavy chains. Their increase in expression has been used as a cardiogenic marker in early embryonic development. ${ }^{12}$ Immunolabeling of alpha- or beta-myosin heavy chain proteins resulted in a nearly identical pattern that only slightly varied in intensity between cells (Figure 1A). The myosin heavy chain isoforms were also found in the freshly isolated ARVM (Figure $3 F)$. Two myosin light chain isoforms have been described as ventricular and atrial-specific proteins in the adult heart $(M L C 2 \mathrm{v}$, MLC2a). ${ }^{13}$ We have found hiPSC-CMs expressing either one of these myosin light chain isoforms or both, to a different extent in the cultures (Figures $1 \mathrm{~B}$ and $4 \mathrm{~A}$ ). As demonstrated by the immunostaining of freshly isolated cells from adult rats, the atrial-specific isoform MLC2a does not occur in ARVM (Figure 3A). Desmin, the predominant intermediate filament protein in striated muscle, has previously been demonstrated in hiPSC-CMs and ARVM in our lab. ${ }^{14,15}$ Vimentin is an intermediate filament protein that is expressed in fetal cardiomyocytes and in non-myocytes in adult

Table 1. Antibodies used in this study.

\begin{tabular}{|c|c|c|}
\hline Target protein & Host & Vendor, clone, article number \\
\hline All actin & Rabbit & Sigma-Aldrich, A2066 \\
\hline Alpha smooth muscle actin (ACTA2) & Mouse & Abcam (Cambridge, UK), clone 1A4, ab7817 \\
\hline Alpha-myosin heavy chain (MYH6) & Rabbit & LifeSpan BioSciences (Seattle, WA, USA), LS-C411049 \\
\hline Alpha-tubulin & Rabbit & GeneTex (Irvine, CA, USA), GTX112141 \\
\hline Beta-catenin & Mouse & Affymetrix eBiosciences (Santa Clara, CA, USA), clone 15B8, 14-2567-80 \\
\hline Beta-myosin heavy chain (MYH7) & Mouse & LifeSpan BioSciences, clone IML-64, LS-C312448 \\
\hline BIN-1/amphiphysin-2 & Mouse & Santa Cruz Biotechnology (Dallas, TX, USA), clone 2F11, sc-23918 \\
\hline Cardiac ankyrin repeat protein (CARP, ANKRD1) & Mouse & Santa Cruz Biotechnology, clone G2, sc-365056 \\
\hline Cardiac troponin-I (cTnI, TNNI3) & Mouse & Thermofisher, clone 4C2, MA1-20116 \\
\hline Cardiac troponin-T (cTnT, TNNT2) & Mouse & Thermofisher, clone 13-11, MA5-12960 \\
\hline Connexin-43 (GJAl) & Rabbit & Abcam, ab11370 \\
\hline Desmin & Mouse & LifeSpan BioSciences, LS-B3122-50 \\
\hline Desmoglein-2 & Mouse & Abcam, clone 3G132, ab14415 \\
\hline Embryonic-heart myomesin (EH-myomesin) & Rabbit & Received as gift 28,29 \\
\hline Junctophilin-2 & Mouse & Santa Cruz Biotechnology, clone H-3, sc-377086 \\
\hline Laminin & Rabbit & Sigma-Aldrich, L9393 \\
\hline M-protein & Mouse & clone AA259, received as gift 29,30 \\
\hline Myomesin (all isoforms) & Mouse & Developmental Studies Hybridoma Bank, University of Iowa, clone B411 \\
\hline Myosin light chain 2v (MLC2v) & Rabbit & Synaptic Systems (Göttingen, Germany), 310203 \\
\hline Myosin light chain-2a (MLC2a) & Mouse & Synaptic Systems, clone 56F5, 311011 \\
\hline $\mathrm{N}$-cadherin & Rabbit & Santa Cruz Biotechnology, sc-7939 \\
\hline Ryanodine receptor & Mouse & Santa Cruz Biotechnology, clone F-1, sc-376507 \\
\hline Sarcomeric alpha-actinin & Mouse & Abcam, clone EA-53 \\
\hline SERCA2a & Mouse & Abcam, clone 2A7-A1, ab2861 \\
\hline Telethonin/titin cap protein (T cap) & Mouse & Santa Cruz Biotechnology, clone G11, sc-25327 \\
\hline Vimentin & Rabbit & Novus Bio (Littleton, CO, USA) / Bio-techne (Minneapolis, MN, USA), NBP1-31327 \\
\hline
\end{tabular}


tissue and was reported to disappear from cardiomyocytes postnatally. ${ }^{16}$ Here we have stained the cells for vimentin, and for myomesin to identify cardiomyocytes (Figure 1C). Vimentin-positive filaments were found in virtually all hiPSC-CMs in the culture, but not in freshly isolated ARVM (Figure 3C). In contrast to vimentin, desmin was observed only in a fraction of the cultured cells (not shown, for quantification see Figure 4A). The Z-line marker protein sarcomeric alpha-actinin was found in all hiPSC-CMs and was organized in sarcomeres (Figure 1D). Microtubules were observed in all hiPSC-CMs in culture (Figure 1D). The distribution pattern of microtubules was not correlated with other cytoskeleton elements as previously observed in cultured adult rat cardiomyocytes. $^{17}$

\section{Cell-cell contact proteins}

We observed prominent labeling of adherens junction, desmosomal and gap junction proteins at cell-cell contacts in the cultured hiPSC-CMs (Figure 2 A,B). Betacatenin-positive junctions occurred at all cell-cell contacts in the monolayer culture (Figure 2A). Connexin-43 was found in the form of gap junction plaques and a perinuclear distribution, as has previously been observed in other cardiomyocyte in vitro models. ${ }^{18}$ Junctions positive for $\mathrm{N}$-cadherin were found at cell-cell contacts partially colocalizing with desmoglein-2, a typical marker of desmosomes ${ }^{19}$ (Figure 2B). Immunostaining freshly isolated ARVM for $\mathrm{N}$-cadherin demonstrated the strictly polarized localization of adherens junctions at the ends of the rod-shaped cells, the intercalated discs (Figure 3G). We also investigated proteins of the so-called costamer complex that provides mechanical anchorage linking Z-discs of sarcomeres via integrins to laminin and other extracellular matrix proteins along the long axis of the cells in tissue. ${ }^{20}$ In the cultured hiPSC-CMs, laminin was found to enclose cultured cardiomyocytes (Figure 1E), but no striated pattern along Z-discs was observed as it is present in the freshly isolated cardiomyocytes immunostained for laminin (Figure $3 \mathrm{H})$.

\section{Calcium-handling proteins}

Calcium-handling proteins are part of the excitation-contraction machinery of cardiomyocytes and are therefore of great interest for disease models and drug screening. Labeling of bridging integrator-1 (bin1), a membrane scaffolding protein that is essential for the formation of T-tubules and their efficient function, ${ }^{21}$ showed bin 1 pos- itive ladders and striation along myofibrils (Figure 2C). Immunostaining of hiPSCCMs for sarcoplasmic reticulum ATPase2a (SERCA2a) showed labeling in virtually all
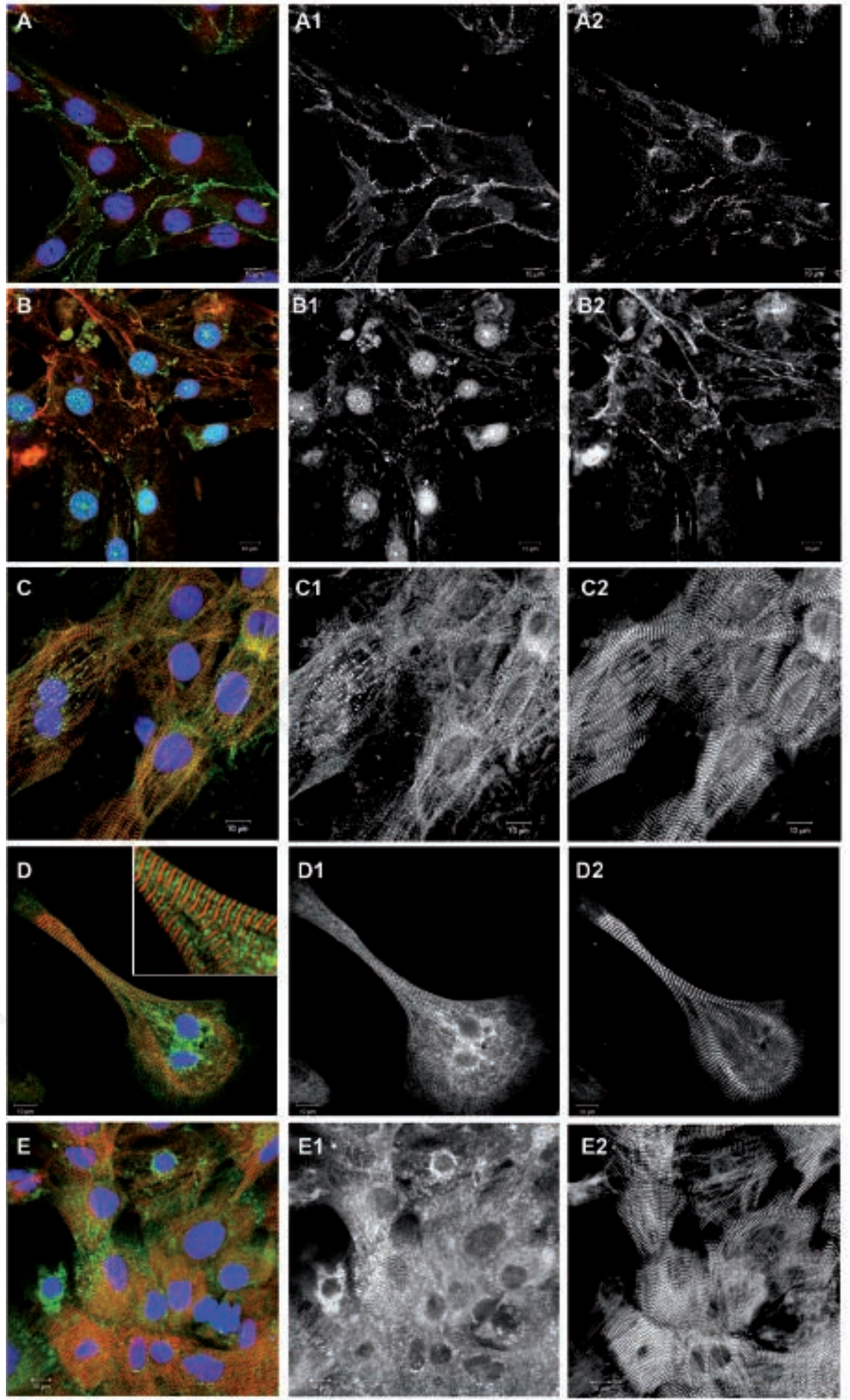

Figure 2. Junctional proteins and proteins involved in calcium handling. Overlay images including DAPI are shown on the left, followed by corresponding green and red channel images in greyscale. A) Cells were immunostained for beta-catenin (green), connexin-43 (red) and DNA (blue). B) Cells were immunostained for $\mathrm{N}$-cadherin (green), desmoglein2 (red) and DNA (blue). C) Cells were immunostained for BIN-1/amphiphysin-2 (green) and EH-myomesin (red). D) Cells were immunostained for SERCA-2a (green), EHmyomesin (red) and DNA (blue). E) Cells were immunostained for ryanodine receptors (green), EH-myomesin (red) and DNA (blue). 
(RyR) showed the protein in all cells, although the level of organization differed in the cell population (Figure 2E). The immunostaining of freshly isolated ARVM for junctophilin-2, a protein that is essential for the structural organization of the above mentioned sarcoplasmic reticulum/ryanodine-complex of calcium-handling proteins, ${ }^{22}$ showed a striated pattern along the long axis of the cells (Figure $3 \mathrm{H}$ ).

\section{Developmentally regulated proteins}

In the following, we report about sarcomeric proteins that are known to undergo an isoform switch from fetal to the adult human myocardium or to show increasing levels of structural organization during development of the heart. We have investigated immunolabeled hiPSC-CMs (Figure 5 ) and performed counting of the observed phenotypes (Figure 4A). Telethonin (also known as T-cap) was described as a signaling and mechano-sensitive element in the heart. ${ }^{23,24}$ We have found a generally intense telethonin labeling of hiPSC-CMs in the 10day old culture, although a sarcomeric striation pattern in the telethonin-channel was only visible in few places (Figures 4A and $5 \mathrm{~A}$ ) that included myofibrils as demonstrated by the actin (Figure 5 A2) or myomesin labeling (Figure 1B). Cardiac ankyrin repeat protein (CARP) (also known as cardiac-adriamycin-responsive protein or ankyrin repeat domain-1, ANKRD1) was found in the culture and showed a striation pattern in a sub-population (Figures 4A and 5B). Additionally, a nuclear localization of CARP in hiPSC-CMs has been noted (Figure $5 \mathrm{~B} 1$ ), as it has been described previously in primary cardiomyocytes. ${ }^{25,26}$ In the freshly isolated adult cardiomyocytes, CARP was observed in all cells showing a distinct I-band striation pattern (Figure 3D). Troponin-I isoforms have been reported to represent good markers for cardiomyocyte maturation because of the switch that occurs during development from the fetal form of slow skeletal TnI to the adult cardiac TnI. ${ }^{27}$ We have used an antibody for the adult cardiac troponin-I isoform (Table 1) and found a patchy expression in the hiPSC-CM culture (Figures 4A and 5C). That image shows a group of cells positive for troponin-I, but also at least one cardiomyocyte in the lower part of the image, that does not show any apparent labeling (Figure $5 \mathrm{C} 1$ ). Cardiac troponin-T was found in all hiPSC-CMs in sarcomeric organization (not shown). Some sarcomeric and cytoskeleton proteins have been observed to re-appear in diseases of the adult heart as part of an adaptation and remodeling process of the myocardium. An

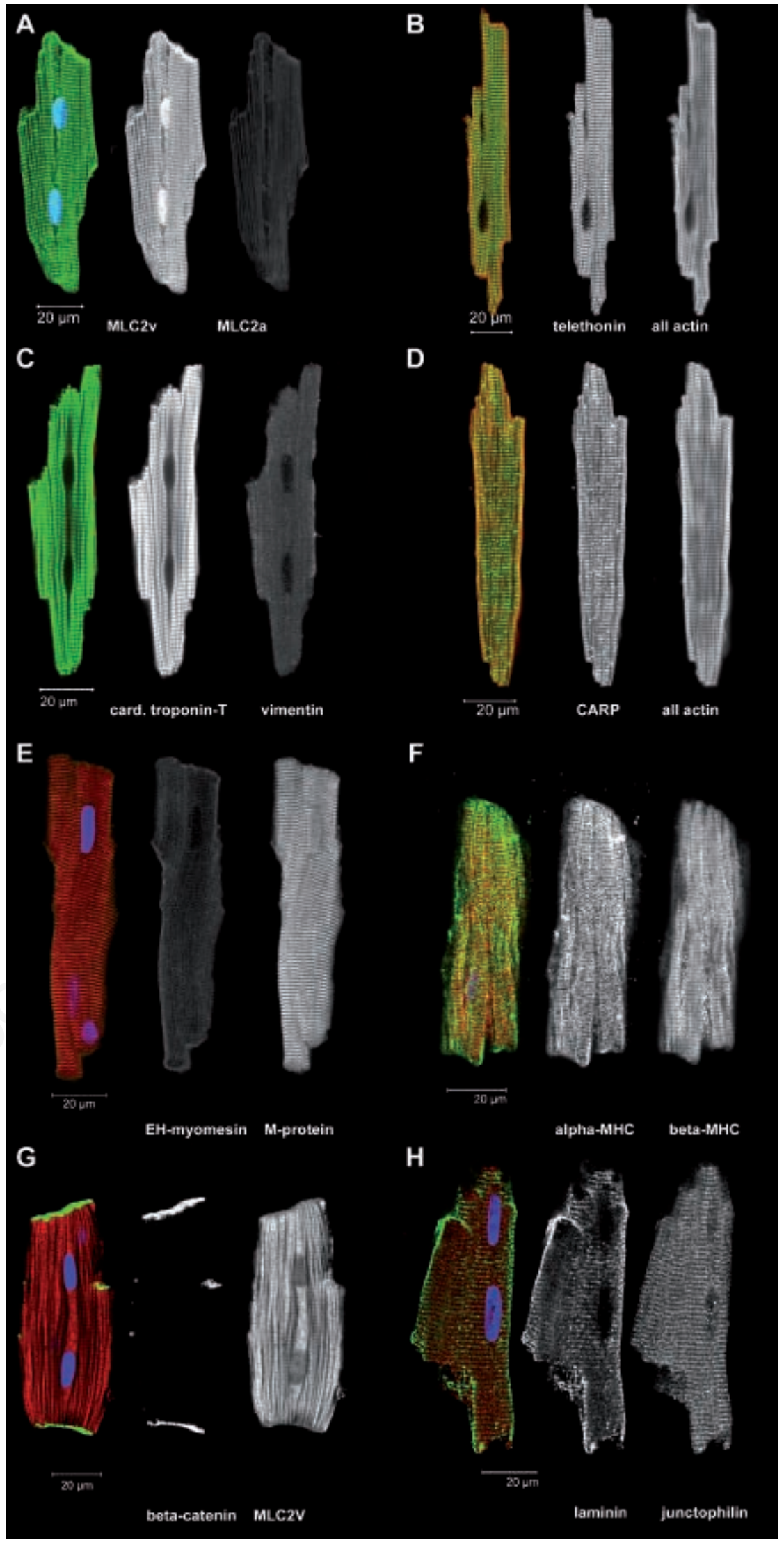

Figure 3. Freshly isolated ARVM were immediately fixed and immunostained for the indicated proteins. A-H) Overlay images of single optical sections are shown in color, some including DAPI staining for DNA in blue, followed by separate images in greyscale with the green channel in the middle and red channel on the right. 
example for this type of proteins is alphasmooth muscle actin, that is only expressed in smooth muscle cells of the vasculature in the normal adult heart, but is found in the myocardium in patients with pathological cardiac hypertrophy. ${ }^{28}$ In the hiPSC-CMS we found a patchy distribution of cells expressing alpha-smooth muscle actin (Figures 4A and 5D). Cardiomyocytes were identified by sarcomeric striation in the allactin image (Figure 5 D2).

\section{Freshly isolated ventricular rat car- diomyocytes}

Freshly isolated ventricular cardiomyocytes (ARVM) from healthy adult rats provide bona fide examples for the fully differentiated endpoint of cytoskeleton and sarcomeric development in the mammalian heart. We have used ARVM for immunocytochemistry of selected proteins that had been previously investigated in the cultured hiPSC-CMs (Figure 3). The same antibodies were used as for the hiPSC-CMs. Generally, immunolabeling was uniform in the population of isolated ARVM unlike the patchy, cell-autonomous distribution seen for some proteins in the cultured hiPSCCMs. The results confirmed the specificity of the myosin light chain $2 \mathrm{a}$ antibodies that do not label ARVM (Figure 3A). The labeling of telethonin showed a well-organized, distinct sarcomeric pattern for the titinbinding protein (Figure 3B). The immunolabeling for cardiac troponin- $\mathrm{T}$ and vimentin confirmed, that vimentin is absent from adult ventricular cardiomyocytes (Figure 3C). The labeling of CARP showed a distinct sarcomeric pattern (Figure 3D). In fetal heart cardiomyocytes, all M-lines contain embryonic-heart myomesin (EHmyomesin), while this isoform is strongly down-regulated after birth. ${ }^{29}$ On the other hand, M-protein is a typical protein of the adult $\mathrm{M}$-line $\mathrm{e}^{30}$ as was confirmed here in ARVM (Figure 3E). EH-myomesin was consistently found in all cultured hiPSCCMs (Figure 2 C,D), but not in freshly isolated ARVM (Figure 3E).

\section{Quantification of immunolabeling and sarcomeric organization}

A manual quantification was performed on populations of cells that showed either incomplete sarcomeric organization as was the case for telethonin (in $9.4 \%$ of all cells organized in sarcomers) and CARP (organized in $32 \%$ ), or showed a patchy distribution of expression such as observed for cardiac troponin-I (found in 19\% cells), alphasmooth muscle actin (in 23\% cells), desmin (in $9.9 \%$ cells), and the atrial/ventricular light chain isoforms (72\% MLC2v vs 25\% MLC2a) (Figure 4A). The quantitative assessment of morphological features can be instrumental to identify the maturation stage of cardiomyocytes ${ }^{7}$ and therefore we evaluated sarcomere length and the overall shape of the cells in hiPSC-CMs compared to ARVM. The analysis of sarcomere length in cultured hiPSC-CMs compared to freshly isolated ARVM showed a significant difference between the two cell types (Figure 4B) with a value of $1.7 \pm 0.13 \mu \mathrm{m}$ for hiPSCCMs and $1.9 \pm 0.09$ for ARVM $(n=40$, $\mathrm{P}<0.001$ ). The form factor, i.e. the ratio of cell length divided by the width, demonstrates the extent of elongation (Figure 4C).
This parameter showed a significant difference between the cells, although there was a considerable amount of variability observed. The ratio was measured as $2.9 \pm 1.7$ for hiPSC-CMs and $5.7 \pm 1.3$ in $\operatorname{ARVM}(\mathrm{n}=17, \mathrm{P}<0.001)$.

\section{Discussion}

As iPSC-derived cells are growing in popularity in recent years for both basic research and drug development, there is a need for a thorough characterization and validation of these new tools. Still, ques-
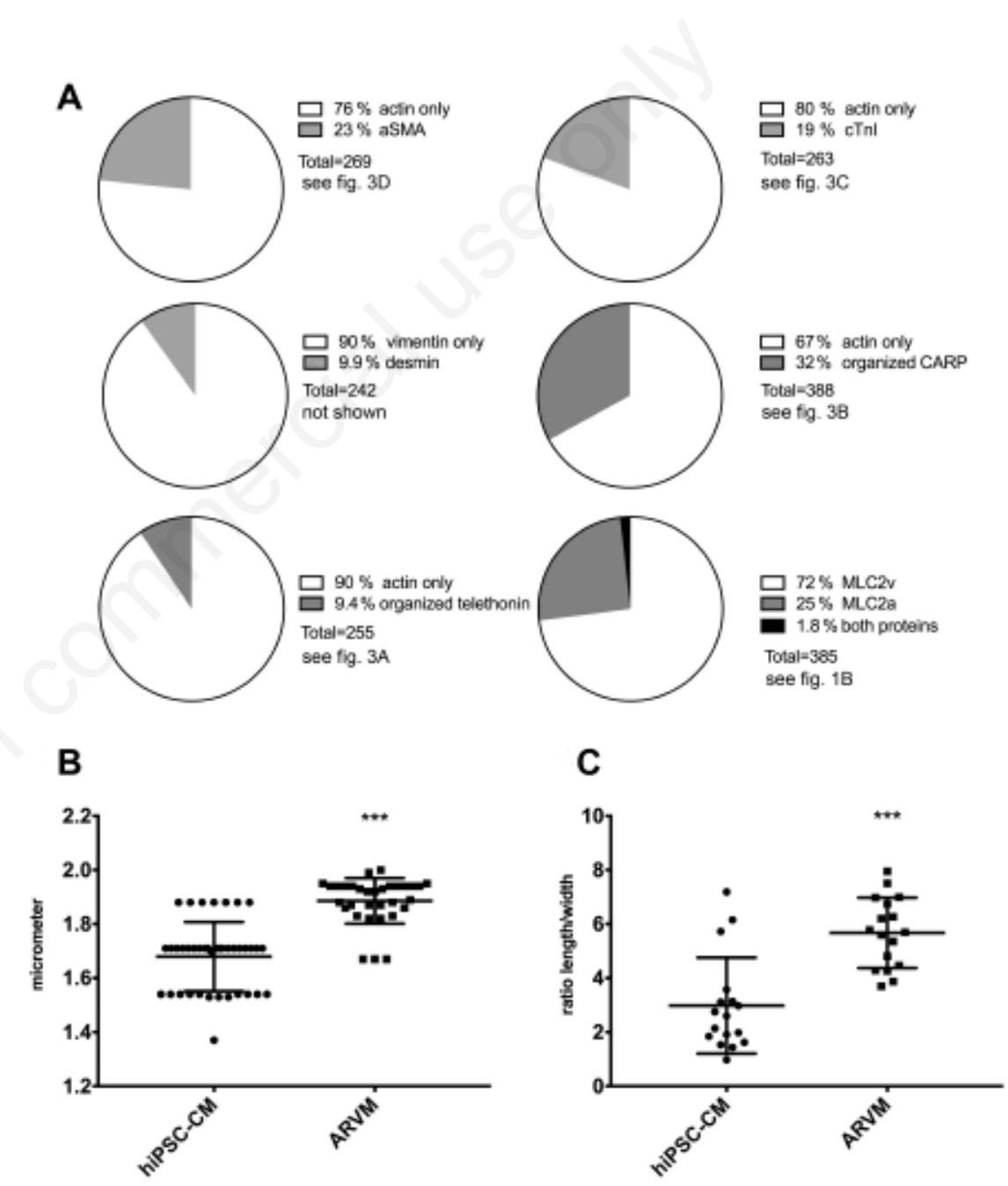

Figure 4 Quantification of immunolabeling and sarcomeric organization found only in a fraction of the cell population, and sarcomere length and form factor in hiPSC-CMs versus adult rat ventricular cardiomyocytes. A) Multiple optical fields from three independent immunocytochemistry experiments were evaluated and results are displayed as parts of a whole (total number of cells evaluated). Actin and vimentin were seen in all cells, while the indicated smaller fraction showed additional proteins/features. B,C) The scatter plots show mean and standard deviation, $\mathrm{n}=\mathrm{A} 40 / \mathrm{B} 17$ cells, ${ }^{* * *} \mathrm{P}<0.001$. B) Comparison of sarcomere lengths in hiPSC-CM versus freshly isolated ARVM. C) Comparison of length-width ratio (form factor) in cultured hiPSC-CM versus freshly isolated ARVM. 
tions are raised about the general limitations of iPSC-derived cardiomyocytes as compared to more traditional models. ${ }^{31}$ In principle, all investigations published so far on the core properties of hiPSC-CMs have found typical features of cardiac muscle. The remaining issues are the immaturity of the cells and the resulting differences in gene expression when compared to the adult human heart. Also, considerable heterogeneity between production lots and within the cell population regarding cardiomyocyte subtypes has been observed. ${ }^{2}$ For complex in vitro model systems with multiple cell types or tissue-like 3D-culture systems and tissue engineering approaches, survival, stability, and development over longer periods of time in culture are rele-
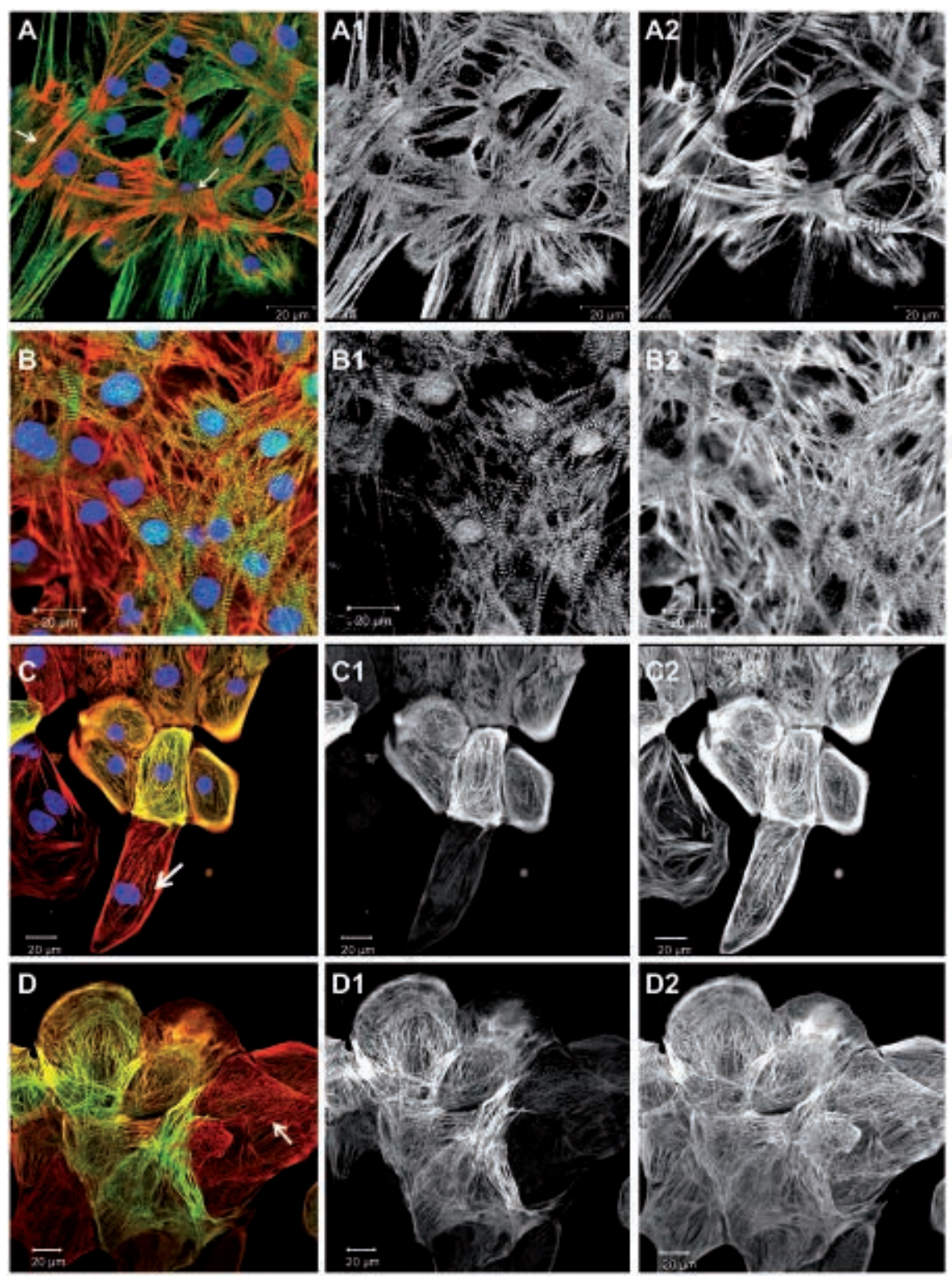

Figure 5. Developmentally regulated sarcomeric proteins. Overlay images including DAPI are shown on the left, followed by corresponding green and red channel images in greyscale. Images A and B were taken at higher magnification to resolve individual sarcomers, while $C$ and $D$ show larger groups of cells, where labelled proteins appeared in a sub-population of cells. A) Cells were immunostained for telethonin (green), all actin (red), and DNA (blue); arrows point at foci of sarcomeric organization. B) Cells were immunostained for CARP (green), all actin (red), and DNA (blue). C) Cells were immunostained for cardiac troponin-I (green), all actin (red), and DNA (blue); the arrow points at a cardiomyocyte negative for troponin-I. All actin labeled all the cells (C2). D) Cells were immunostained for alpha-smooth muscle actin (green) and all actin (red); the arrow points at a cardiomyocyte negative for alpha-smooth muscle actin and showing sarcomeric striation in the actin channel; actin was observed in all cells (D2). vant. ${ }^{14,32}$ These cytoskeleton markers and the corresponding immunoreagents that we have evaluated in this study provide effective tools for future investigations to assess the maturation status of tissue constructs, especially in the field of tissue engineering where the integration of the tissue in a fully developed host environment is crucial. Although a variety of techniques for characterizing hiPSC-CM has already been employed so far, immunocytochemistry provides a rapid and straightforward tool to obtain information both on the protein expression and differentiation level of cellular features.

We have tested proteins that are either known to become organized later in development or represent associated proteins that may serve as signaling or mechano-sensitive elements. The intermediate filament proteins desmin and vimentin are both expressed in the embryonal and postnatal heart, but only desmin remains as the characteristic intermediate protein of both striated and smooth muscle in the adult. ${ }^{16}$ The sarcomeric M-line protein myomesin serves as a bona fide marker for myofibrils and genuine cardiomyocytes in our experiments, although by electron microscopy, electron-dense M-lines as a further sign of maturation have not yet been found in standard cultures of hiPSC-CMs. ${ }^{3}$ Regarding the regulatory myosin light chain proteins it has been reported, that almost all early hiPSC-CM's express MLC2a, and then at later time-points, the MLC2 $\mathrm{v}$ expressing population becomes predominant, although some cells still expressed or co-expressed MLC $2 \mathrm{a}^{13}$ (and personal observations). All hiPSC-CMs were positive for cardiac troponin-T (cTnT) and the labeling was equal in the entire cell population (not shown). Regarding troponin-I, expression from the fetal TNNI1 gene (ssTnI) is completely replaced in the adult heart by TNNI3 $(\mathrm{cTnI}) .{ }^{33}$ In cultured hiPSC-CMs, cTnI labeling demonstrated different levels in the culture with some cells showing no labeling at all, while others are strongly stained. Similarly, CARP labeling showed a patchy distribution, however with a distinctly visible sarcomeric structure in strongly expressing cells. CARP is sensitive to cardiotoxic cancer therapies and it is upregulated in left ventricular heart failure, ${ }^{34}$ but it is also normally expressed in cardiomyocytes. Alpha smooth muscle actin (aSMA) is expressed in the fetal and postnatal heart. ${ }^{28} \mathrm{~A}$ different picture was observed in telethonin-labeled cells. It has been proposed that telethonin (T-cap) is important for the maturation of sarcomeres, for sensing biomechanical stress, and that its 
Table 2. Qualitative summary of protein expression and organization as observed in this study (left column), and notes about the known pattern in the adult human heart as previously reported (right column).

\begin{tabular}{lcc}
\hline Protein & Labeling and organiration in hiPSC-CMs & Presence in normal adult ventricular cardiomyocytes \\
All actin & +++ & Yes \\
Alpha smooth muscle actin & ++ & No \\
\hline Beta-catenin & +++ & Yes \\
BIN-1 & ++ & Yes \\
\hline Cardiac troponin-I & ++ & Yes \\
Cardiac troponin-T & +++ & Yes \\
\hline CARP & ++ & Yes \\
Connexin-43 & +++ & Yes \\
\hline Myomesin & +++ & Yes \\
EH-myomesin & +++ & No \\
\hline M-protein & + & Yes \\
Myosin light chain 2a & ++ & Yes, atrium \\
Myosin light chain 2v & ++ & Yes, ventricle \\
SERCA2a & ++ & Yes \\
Telethonin (T-cap) & + & Yes \\
Vimentin & ++ & No
\end{tabular}

expression rapidly reacts to stimuli that lead to muscular atrophy or growth. 32,35 Although the signal was generally intense in all hiPSC-CMs, a distinct sarcomeric pattern was visible only in some places, suggesting a robust expression of telethonin but incomplete organization of the protein into sarcomeres. These cytoskeleton proteins discussed above may become instrumental in the assessment of maturation in cardiac in vitro models, since these proteins demonstrate stages of a progression. Calcium handling proteins such as the sodium-calcium exchanger and ryanodine receptor have been detected in hiPSC-CMs previously by western blotting in our lab. ${ }^{14}$

Bin-1 is a membrane scaffolding protein in cardiomyocytes involved in the trafficking of calcium-handling proteins to the $\mathrm{T}$ tubules via microtubules. ${ }^{21}$ Its localization in an I band-associated pattern in the hiPSC-CMs suggests that it is either forming early T-tubules or a precursor protein complex. Intercalated disc proteins in the adult tissue are restricted to the ends of the cylindrical cardiomyocytes. ${ }^{36}$ The pattern of adherens and gap junctions as well as desmosomes observed here in hiPSC-CMs appeared similar compared to other culture models of cultured cardiomyocytes. ${ }^{18}$ It is therefore unlikely, that cell contact proteins are the first choice for measuring maturation of cardiomyocyte models unless the geometry of the cells could be modified to such extent that the strict polarization seen in tissue reappears. In addition to immunocytochemistry, additional methods such as evaluation of morphological parameters, protein biochemistry and gene expression studies will complete the toolbox for the development of improved in vitro models.

In conclusion, all proteins that are known to be expressed in primary cardiomyocytes have been found in the hiPSC-CMs on day 10 in culture, identifying them as genuine cardiomyocytes. However, the expression pattern and patchy distribution of some proteins suggest that the cell population is still developing. A summary of the qualitative assessment of expression and organization of the investigated proteins is presented in Table 2.

\section{References}

1. Takahashi K, Yamanaka S. Induction of pluripotent stem cells from mouse embryonic and adult fibroblast cultures by defined factors. Cell 2006;126:66376.

2. Denning C, Borgdorff V, Crutchley J, Firth KSA, George V, Kalra S, et al. Cardiomyocytes from human pluripotent stem cells: From laboratory curiosity to industrial biomedical platform. Biochim Biophys Acta 2015;1863:1728-48.

3. Yang $X$, Pabon L, Murry CE. Engineering adolescence: maturation of human pluripotent stem cell-derived cardiomyocytes. Circ Res 2014;114: 511-23.

4. Burridge PW, Matsa E, Shukla P, Lin ZC, Churko JM, Ebert AD, et al.
Chemically defined generation of human cardiomyocytes. Nat Methods 2014;11:855-60.

5. Breckwoldt K, Weinberger F, Eschenhagen T. Heart regeneration. Biochim Biophys Acta 2016;1863:1749-59.

6. Uosaki H, Cahan P, Lee DI, Wang S, Miyamoto M, Fernandez L, et al. Transcriptional Landscape of Cardiomyocyte Maturation. Cell Reports 2015;13:1705-16.

7. Veerman CC, Kosmidis G, Mummery CL, Casini S, Verkerk AO, Bellin M. Immaturity of Human stem-cell-derived cardiomyocytes in culture: Fatal flaw or soluble problem? Stem Cells Dev 2015;24:1035-52.

8. Giraud M-N, Flück M, Zuppinger C, Suter TM. Expressional reprogramming of survival pathways in rat cardiocytes by neuregulin-1beta. J Appl Physiol 2005;99:313-22.

9. Shy D, Gillet L, Ogrodnik J, Albesa M, Verkerk AO, Wolswinkel R, et al. PDZ domain-binding motif regulates cardiomyocyte compartment-specific NaV1.5 channel expression and function. Circulation 2014;130:147-60.

10. Agarkova I, Perriard J-C. The M-band: an elastic web that crosslinks thick filaments in the center of the sarcomere. Trends Cell Biol 2005;15:477-85.

11. Grove BK, Kurer V, Lehner C, Doetschman TC, Perriard JC, Eppenberger HM. A new 185,000-dalton skeletal muscle protein detected by monoclonal antibodies. J Cell Biol 
1984;98:518-24.

12. Lundy SD, Zhu W-Z, Regnier M, Laflamme MA. Structural and functional maturation of cardiomyocytes derived from human pluripotent stem cells. Stem Cells Dev 2013;22:19912002.

13. Kamakura T, Makiyama T, Sasaki K, Yoshida Y, Wuriyanghai Y, Chen J, et al. Ultrastructural maturation of humaninduced pluripotent stem cell-derived cardiomyocytes in a long-term culture. Circ J 2013;77:1307-14.

14. Beauchamp P, Moritz W, Kelm JM, Ullrich ND, Agarkova I, Anson B, et al. Development and characterization of a scaffold-free 3D spheroid model of iPSC-derived human cardiomyocytes. Tissue Eng Part C Methods 2015;21:852-61.

15. Chiusa M, Hool S-L, Truetsch P, Djafarzadeh S, Jakob SM, Seifriz F, et al. Cancer therapy modulates VEGF signaling and viability in adult rat cardiac microvascular endothelial cells and cardiomyocytes. J Mol Cell Cardiol 2012;52:1164-75.

16. Kim HD. Expression of intermediate filament desmin and vimentin in the human fetal heart. Anat Rec 1996;246:271-8.

17. Pentassuglia L, Timolati F, Seifriz F, Abudukadier K, Suter TM, Zuppinger C. Inhibition of ErbB2/neuregulin signaling augments paclitaxel-induced cardiotoxicity in adult ventricular myocytes. Exp Cell Res 2007;313: 1588-601.

18. Zuppinger C, Schaub MC, Eppenberger HM. Dynamics of early contact formation in cultured adult rat cardiomyocytes studied by $\mathrm{N}$-cadherin fused to green fluorescent protein. J Mol Cell Cardiol 2000;32:539-55.

19. Vermij, Abriel H, van Veen TA. Refining the molecular organization of the cardiac intercalated disc. Cardiovasc Res 2016;113:1-32.

20. Brancaccio M, Hirsch E, Notte A,
Selvetella G, Lembo G, Tarone G. Integrin signalling: the tug-of-war in heart hypertrophy. Cardiovasc Res 2006;70(3):422-33.

21. Hong T, Yang H, Zhang S-S, Cho HC, Kalashnikova M, Sun B, et al. Cardiac BIN1 folds T-tubule membrane, controlling ion flux and limiting arrhythmia. Nature Research 2014;20(6):62432.

22. Munro ML, Jayasinghe ID, Wang Q, Quick A, Wang W, Baddeley D, et al. Junctophilin-2 in the nanoscale organisation and functional signalling of ryanodine receptor clusters in cardiomyocytes. J Cell Sci 2016;129:4388-98.

23. Ibrahim M, Siedlecka U, Buyandelger B, Harada M, Rao C, Moshkov A, et al. A critical role for Telethonin in regulating t-tubule structure and function in the mammalian heart. Hum Mol Genet 2013;22:372-83.

24. Knöll R, Linke WA, Zou P, Miocic S, Kostin S, Buyandelger B, et al. Telethonin deficiency is associated with maladaptation to biomechanical stress in the mammalian heart. Circ Res 2011;109:758-69.

25. Bang ML, Mudry RE, McElhinny AS, Trombitás K, Geach AJ, Yamasaki R, et al. Myopalladin, a novel 145-kilodalton sarcomeric protein with multiple roles in Z-disc and I-band protein assemblies. J Cell Biol 2001;153:413-27.

26. Zhong L, Chiusa M, Cadar A, Lin A, Samaras S, Davidson JM, et al. Targeted inhibition of ANKRD1 disrupts sarcomeric ERK-GATA4 signal transduction and abrogates phenylephrine-induced cardiomyocyte hypertrophy. Cardiovasc Res 2015;106:26171

27. Bedada FB, Chan SS-K, Metzger SK, Zhang L, Zhang J, Garry DJ, et al. Acquisition of a quantitative, stoichiometrically conserved ratiometric marker of maturation status in stem cellderived cardiac myocytes. Stem Cell Rep 2014;3:594-605.
28. Black FM, Packer SE, Parker TG, Michael LH, Roberts R, Schwartz RJ, et al. The vascular smooth muscle alphaactin gene is reactivated during cardiac hypertrophy provoked by load. J Clin Invest 1991;88:1581-8.

29. Agarkova I, Auerbach D, Ehler E, Perriard JC. A novel marker for vertebrate embryonic heart, the EHmyomesin isoform. J Biol Chem 2000; 275:10256-64.

30. Vinkemeier U, Obermann W, Weber K, Fürst DO. The globular head domain of titin extends into the center of the sarcomeric $\mathrm{M}$ band. cDNA cloning, epitope mapping and immunoelectron microscopy of two titin-associated proteins. J Cell Sci 1993;106:319-30.

31. Knollmann BC. Induced pluripotent stem cell-derived cardiomyocytes: boutique science or valuable arrhythmia model? Circ Res 2013 Mar 15;112:96976

32. Ehler E. Cardiac cytoarchitecture - why the "hardware" is important for heart function! Biochim Biophys Acta 2016;1863:1857-63

33. Jin J-P, Zhang Z, Bautista JA. Isoform diversity, regulation, and functional adaptation of troponin and calponin. Crit Rev Eukaryot Gene Expr 2008;18:93-124.

34. Zolk O, Frohme M, Maurer A, Kluxen F-W, Hentsch B, Zubakov D, et al. Cardiac ankyrin repeat protein, a negative regulator of cardiac gene expression, is augmented in human heart failure. Biochem Biophys Res Commun 2002;293:1377-82.

35. Mason P, Bayol S, Loughna PT. The novel sarcomeric protein telethonin exhibits developmental and functional regulation. Biochem Biophys Res Commun 1999;257:699-703.

36. Hirschy A, Schatzmann F, Ehler E, Perriard J-C. Establishment of cardiac cytoarchitecture in the developing mouse heart. Dev Biol 2006;289:430-41. 